\title{
Otimização do Passo do Algoritmo LMS na Estimação de Canais de HF
}

\author{
Claudio Canto dos Santos, Juraci Ferreira Galdino e Ernesto Leite Pinto
}

\begin{abstract}
Resumo-Este artigo apresenta uma análise de desempenho do algoritmo LMS na estimação de canais de HF, estendendo para este contexto o trabalho feito em [1] e obtendo alguns novos resultados de interesse. Em particular, obtém-se uma expressão analítica aproximada para o valor do passo ótimo, no sentido da minimização do erro quadrático médio na estimação dos coeficientes do canal, em condições de regime estacionário. Diversos resultados numéricos obtidos por análise e simulação são apresentados, validando as análises aqui desenvolvidas e ilustrando sua importância.
\end{abstract}

Palavras-Chave- estimação de canal, comunicações em HF, algoritmo LMS, otimização de passo, MSWE em regime permanente.

Abstract-A performance analysis of the LMS algorithm in the estimation of $\mathrm{HF}$ channels is addressed in this article, extending to this context the work in [1] and obtaining some new contributions of interest. In particular, an approximate analytical expression is derived for the optimum value of the LMS step-size parameter, in the sense of minimizing the mean square error in the estimation of channel gains, under steady-state conditions. Several numerical results of performance evaluation by analysis and simulation are provided, in order to validate the analysis herein presented and illustrate its importance.

Keywords - channel estimation, HF communications, LMS algorithm, step-size optimization, steady-state MSWE.

\section{INTRODUÇÃO}

A transmissão via rádio na faixa de HF (High Frequency) é de grande interesse para cobrir longas distâncias, devido aos diferentes modos de propagação presentes. O sinal que chega ao receptor percorre diversos caminhos oriundos de múltiplas reflexões, na ionosfera ou mesmo no solo. Em cada componente multipercurso ocorre desvanecimento variante no tempo, devido às irregularidades na ionização, produzindo o efeito de espalhamento Doppler [2].

$\mathrm{O}$ espalhamento temporal entre as componentes multipercurso provoca interferência entre símbolos (IES), levando à necessidade de técnicas eficazes de equalização. A busca destas técnicas é um campo de pesquisa bastante atual, devido ao interesse no desenvolvimento de sistemas de transmissão em HF com eficiência espectral cada vez mais elevada [3].

Boa parte dos equalizadores atualmente em uso ou sob investigação se caracteriza pelo emprego de estimativas da resposta ao impulso do canal em tempo discreto, empregando modelos FIR [4]. A escolha do algoritmo de estimação pode ter um impacto muito significativo no desempenho e complexidade destes equalizadores, especialmente quando aplicados a canais variantes no tempo, como é o caso dos canais de HF.

Claudio Canto dos Santos, Juraci Ferreira Galdino e Ernesto Leite Pinto Departamento de Engenharia Eletrica, Instituto Militar de Engenharia, Rio de Janeiro, Brasil, E-mails: claudio.canto@hotmail.com, ernesto@ime.eb.br, galdino@ime.eb.br.
O algoritmo LMS é particularmente interessante para esta aplicação, por apresentar complexidade computacional muito baixa e boa velocidade de convergência quando a seqüência de entrada do filtro adaptativo é um processo estocástico branco, o que é usual no contexto de estimação de canais.

A efetiva exploração das vantagens deste algoritmo, no entanto, depende de uma criteriosa escolha do seu passo de adaptação, o qual tem impacto direto sobre a estabilidade, as características de convergência e o nível de erro quadrático médio alcançado em regime permanente [5].

Em [1] é feita uma análise de desempenho do algoritmo LMS na estimação de canais variantes no tempo modelados como WSS-US (WideSense Stationary - Uncorrelated Scattering). Lá são obtidas expressões analíticas para o erro quadrático médio nos coeficientes (MSWE, de Mean Square Weight estimation Error) em regime permanente, em função do valor do passo e de parâmetros típicos de sistemas de comunicação. Além disso, são apresentadas aproximações analíticas para o valor ótimo do passo no sentido de reduzir o MSWE de regime permanente, considerando-se alguns modelos de espectro Doppler, dentre os quais o modelo de Jakes.

O presente trabalho estende a análise apresentanda em [1] para o contexto de canais de HF. Adota-se aqui um modelo específico de espalhamento Doppler usualmente associado à propagação ionosférica em HF [2]. Além da otimização do passo, também se analisa o aumento do MSWE devido à limitação do número de coeficientes do modelo em tempo discreto (filtro FIR) utilizado para estimação de canal, levandose em conta o efeito dos filtros de transmissão e recepção.

O restante deste texto está organizado em quatro seções. $\mathrm{Na}$ Seção II apresenta-se os modelos adotados para o sistemas de transmissão e para o canal de HF. Na Seção III é revista brevemente e estendida para canais de HF a análise apresentada em [1]. Nesta mesma seção se analisa a otimização do passo do LMS e o efeito da quantidade reduzida de coeficientes do filtro de estimação sobre o MSWE. Na Seção IV diversos resultados numéricos de aplicação das análises aqui realizadas são comparados com resultados de simulações. Por fim, na Seção V são sumarizadas as conclusões do trabalho.

\section{Modelo do Sistema de Comunicações}

O modelo do sistema de comunicação em banda básica aqui considerado é mostrado na Figura 1.

A envoltória complexa do sinal na saída do filtro de transmissão é dada por:

$$
x(t)=\sum_{k} s_{k} g_{T}\left(t-k T_{s}\right),
$$




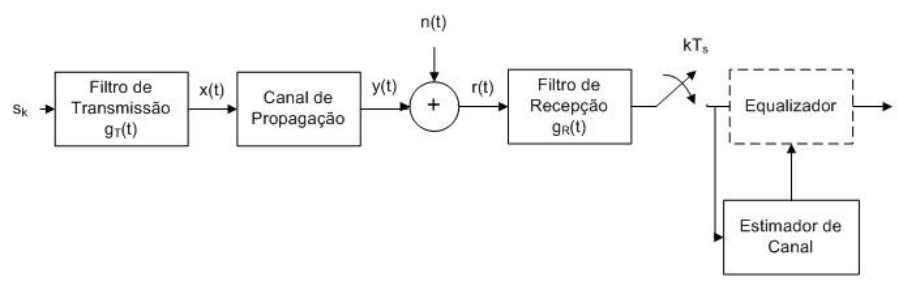

Fig. 1. Modelo do sistema de comunicação.

onde $\left\{s_{k}\right\}$ é a seqüência de símbolos da modulação, $g_{T}(t)$ é a resposta ao impulso deste filtro e $T_{s}$ é o intervalo de símbolo. Admite-se que os símbolos transmitidos são descorrelacionados e de média nula.

O canal de propagação é modelado como um filtro linear com $J$ coeficientes variantes no tempo. O sinal na entrada do filtro de recepção é da forma:

$$
r(t)=\sum_{j=0}^{J-1} h_{j}(t) x\left(t-\tau_{j}\right)+n(t)
$$

onde $\left\{h_{j}(t)\right\}$ e $\left\{\tau_{j}\right\}$ são, respectivamente, os ganhos e atrasos do canal, e $n(t)$ é um ruído aditivo Gaussiano branco de média nula, estatisticamente independente do sinal transmitido e do canal de propagação.

Admite-se que o filtro de recepção é casado, com resposta ao impulso $g_{R}(t)$ do tipo raiz quadrada de cosseno levantado. A saída deste filtro é amostrada à taxa de símbolos e em seguida usada para os processamentos de estimação e equalização de canal.

Os canais de propagação em HF são usualmente modelados em banda básica como canais WSS-US (de Wide Sense Stationary - Uncorrelated Scattering), com dois valores de retardos $(J=2)$. Os ganhos $h_{j}(t)$ são modelados como processos estocásticos Gaussianos estacionários em sentido amplo, de média nula e espectro de potência com formato Gaussiano, centrado na origem.

O modelo de espectro Doppler normalizado é dado por

$$
S(\lambda)=\frac{1}{\sqrt{\pi / 2} \cdot B_{d}} e^{-\frac{2 \lambda^{2}}{B_{d}^{2}}}
$$

em que $B_{d}$ é o espalhamento Doppler.

No que diz respeito ao efeito de espalhamento de retardos, em geral se admite que os dois ganhos do canal têm mesma potência, de modo que diferentes níveis de intensidade deste efeito ficam modelados pelo valor do retardo (relativo) do segundo raio, que será aqui denotado por $\tau_{s}$.

Para realização de avaliações de desempenho em canais de HF alguns modelos específicos foram estabelecidos pelo grupo de comunicações via rádio da International Telecommuncations Union (ITU-R, sucessor do CCIR). A norma ITU-R F.520 previa três modelos de canal, os quais são bastante utilizados até hoje, sendo conhecidos como "canais CCIR"1. Estes modelos são usados no presente trabalho, e

\footnotetext{
${ }^{1}$ Esta norma foi substituída no ano de 2000 pela ITU-R F.1487, que prevê dez modelos de canal para testes, incluindo os três citados da norma F.520.
}

estão especificados na tabela Tabela I.

TABELA I

ParÂMETRos dos CANAIS DE TESTE UTILIZAdos

\begin{tabular}{|c|l|r|rr|}
\hline Norma & Canal & $\tau_{s}$ & $B_{d}$ \\
\hline \multirow{3}{*}{ F.520 } & ITU-R bom & $0,5 \mathrm{~ms}$ & $0,1 \mathrm{~Hz}$ \\
\cline { 2 - 4 } & ITU-R moderado & $1 \mathrm{~ms}$ & $0,5 \mathrm{~Hz}$ \\
\cline { 2 - 5 } & ITU-R pobre & $2 \mathrm{~ms}$ & $1 \mathrm{~Hz}$ \\
\hline
\end{tabular}

\section{ANÁlise DE DESEMPENHO}

É apresentada em [1] uma análise matemática do MSWE (Mean Square Weight estimation Error) produzido pelo algoritmo LMS na estimação de canais WSS-US. O MSWE, denotado por $D(n, \mu)$, é definido como

$$
D(n, \mu) \equiv E\left[\|\mathbf{w}(n)-\hat{\mathbf{w}}(n, \mu)\|^{2}\right]
$$

onde $\mathbf{w}(n)$ é o vetor de coeficientes da resposta ao impulso do canal em tempo discreto e $\hat{\mathbf{w}}(n, \mu)$ é o seu estimador ${ }^{2}$.

Sob condições de regime estacionário, mostra-se em [1] que o MSWE pode ser dado em função do passo $\mu$ por

$D(\mu)=\frac{\mu \sigma_{n}^{2} M+2 \sigma_{w}^{2} \sum_{l=0}^{\infty}\left(1-\mu \sigma_{s}^{2}\right)^{l}[R(l)-R(l+1)]}{\left(2-\mu M \sigma_{s}^{2}\right)}$, onde $M$ é o número de coeficientes da resposta ao impulso do canal, $R(l) \equiv R\left(l T_{s}\right)$, sendo $R($.$) a função autocorrelação$ normalizada dos coeficientes do canal, $\sigma_{w}^{2}$ é a soma das variâncias destes coeficientes, $\sigma_{s}^{2}$ é a variância dos símbolos e $\sigma_{n}^{2}$ é a variância do ruído.

\section{A. Otimização do Passo}

Para fins de otimização do passo, reescreve-se aqui a expressão de $D(\mu)$ acima como:

$$
D(\mu)=\frac{1}{\left(2-\mu M \sigma_{s}^{2}\right)}\left\{\mu \sigma_{n}^{2} M+2 \sigma_{w}^{2} \frac{\left(1-\mu \sigma_{s}^{2} P\right)}{\left(1-\mu \sigma_{s}^{2}\right)}\right\},
$$

com

$$
P \equiv \sum_{l=0}^{\infty}\left(1-\mu \sigma_{s}^{2}\right)^{l} R(l) .
$$

Expressando-se $R(l)$ através do espectro Doppler normalizado $S(\lambda)$, e após algumas manipulações simples, obtém-se:

$$
P=\int_{-\infty}^{\infty} S(\lambda) p(\lambda) d \lambda
$$

sendo

$$
p(\lambda)=\frac{1}{1-\left(1-\mu \sigma_{s}^{2}\right) e^{j 2 \pi \lambda T_{s}}} .
$$

Como o valor do espalhamento Doppler é usualmente bem menor que a taxa de símbolos, utiliza-se uma aproximação de segunda ordem para $p(\lambda)$, no cálculo da integral da expressão (7). Levando o resultado na expressão (5), obtém-se uma

\footnotetext{
${ }^{2}$ A modelagem do sistema de comunicações adotada em [1] é semelhante à empregada aqui, exceto por uma única diferença significativa, que é discutida na subseção III-B.
} 
aproximação conveniente para $D(\mu)$, em termos do momento de segunda ordem de $S(\lambda)^{3}$. No caso em foco, este momento é igual a $B_{d}^{2} / 4$, como se pode ver a partir de (3).

Calculando a derivada parcial desta expressão aproximada de $D(\mu)$ com relação a $\mu$ e igualando o resultado a zero, verifica-se que o passo ótimo, no sentido da minimização do MSWE, pode ser obtido de forma aproximada resolvendo-se a seguinte equação:

$$
A_{3} \mu^{3}+A_{2} \mu^{2}+A_{1} \mu+A_{0}=0,
$$

cujos coeficientes são dados por:

$$
\begin{aligned}
& A_{3}=\sigma_{n}^{2} M \sigma_{s}^{4} ; \\
& A_{2}=-2 \sigma_{w}^{2} \sigma_{s}^{4} K M \\
& A_{1}=2 \sigma_{w}^{2} K \sigma_{s}^{2}(3 M+1) \\
& A_{0}=-8 \sigma_{w}^{2} K,
\end{aligned}
$$

onde $K=\left(\pi T_{s} B_{d}\right)^{2}$.

Cabe notar que a Equação (9) tem apenas uma solução real e positiva, que é o valor aproximado do passo ótimo. Esta solução não é apresentada aqui, para fins de concisão.

É válido observar também que a metodologia acima descrita para cálculo aproximado de $D(\mu)$ e otimização do passo pode ser aplicada a diferentes modelos de espalhamento Doppler.

\section{B. Efeito dos Filtros de Transmissão e Recepção no MSWE}

$\mathrm{Na}$ análise desenvolvida em [1] admite-se que o canal é modelado com retardos múltiplos do intervalo de símbolo. Nas condições de transmissão consideradas no presente trabalho esta hipótese nem sempre é válida, o que leva à necessidade de se considerar o efeito conjunto do canal de propagação, dos filtros de transmissão e recepção e da taxa de amostragem no estabelecimento do modelo de canal em tempo discreto a ser utilizado para equalização, e na avaliação do MSWE.

Cabe notar inicialmente que os filtros de transmissão e de recepção se caracterizam por ter resposta ao impulso com decaimento assintótico com o tempo, dando origem a uma resposta ao impulso conjunta do canal de mesma natureza. No entanto, para evitar o aumento de complexidade do receptor, o estimador de canal deve muitas vezes empregar um modelo FIR com uma quantidade reduzida de coeficientes. Ocorre assim um "truncamento"no modelo de canal, que dá origem a um aumento no MSWE aqui denominado de erro de truncamento e denotado por $\epsilon$.

Para se avaliar analiticamente este erro de truncamento, considera-se que a associação em cascata do filtro de recepção e do canal pode ser alterada, obtendo-se o modelo aproximado mostrado na Figura 2.

Em [7] verificou-se que esta aproximação é satisfatória quando a resposta ao impulso do canal de propagação pode ser considerada aproximadamente invariante em intervalos de tempo de ordem da duração efetiva ${ }^{4}$ da resposta ao impulso do

\footnotetext{
${ }^{3}$ Os detalhes destas manipulações encontram-se em [6].

${ }^{4}$ Por duração efetiva de um pulso de duração ilimitada entende-se a largura de uma janela de tempo na qual estão contidos $99 \%$ de sua energia.
}

filtro de recepção. A análise dos parâmetros dos sistemas de transmissão e dos modelos de canal mostra ser esta condição plenamente atendida nas condições abordadas no presente trabalho, que estão detalhadas na seção IV.

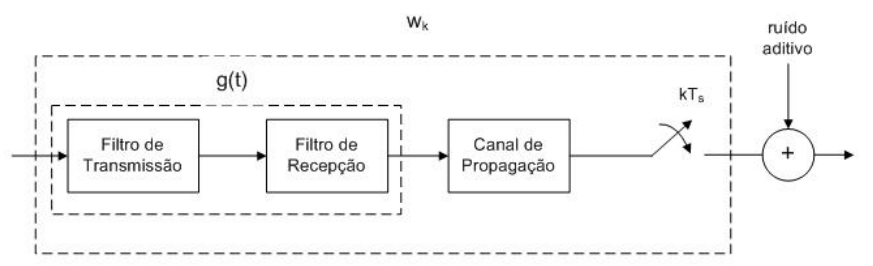

Fig. 2. Modelo aproximado para associação em cascata de filtros e canal.

Note-se agora, com base na Figura 2, que a resposta ao impulso conjunta do canal é uma soma de impulsos com atrasos múltiplos do intervalo de símbolo. Estes impulsos são ponderados por coeficientes (pesos) determinados pelos ganhos do canal de propagação e pela resposta ao impulso dos filtros de transmissão e recepção.

Denota-se por $w(m, n)$ um destes coeficientes (arbitrário), o qual pode ser associado à resposta observada no instante $t=n T_{s}$ (na saída do amostrador) a um impulso aplicado em $t=(n-m) T_{s}$. Com base na aproximação mostrada na Figura 2 verifica-se que $w(m, n)$ pode ser expresso por:

$$
w(m, n)=\sum_{j=0}^{J-1} h_{j}\left(n T_{s}\right) g\left(m T_{s}-\tau_{j}\right)
$$

onde $g(t) \equiv g_{T}(t) * g_{R}(t)$ é um pulso do tipo cosseno levantado.

Como os ganhos do canal de propagação são por hipótese estacionários em sentido amplo, mutuamente descorrelacionados e de média nula, a potência (variância) do coeficiente $w(m, n)$ não depende de $n$ e pode ser dada por

$$
\sigma_{m}^{2} \equiv E\left[|w(m, n)|^{2}\right]=\sum_{j=0}^{J-1} \sigma_{j}^{2} g^{2}\left(m T_{s}-\tau_{j}\right),
$$

onde $\sigma_{j}^{2}$ é a potência do $j$-ésimo ganho do modelo de canal de propagação.

Cabe notar neste ponto que, como $g(t)$ é ilimitado no tempo, há em princípio um número infinito de coeficientes $w(m, n)$ com variância $\sigma_{m}^{2}$ não nula. Para estabelecimento de um modelo em tempo discreto é interessante escolher o índice do primeiro coeficiente (aqui denotado por $m_{0}$ ) de modo a maximizar a soma das variâncias dos coeficientes.

Admitindo que o canal de propagação e a resposta em cosseno levantado $g(t)$ são normalizados, o erro de truncamento $\epsilon$ correspondente a um modeldo com $M$ coeficientes fica dado por:

$$
\epsilon=1-\sum_{m=m_{0}}^{m_{0}+M-1} \sigma_{m}^{2}
$$




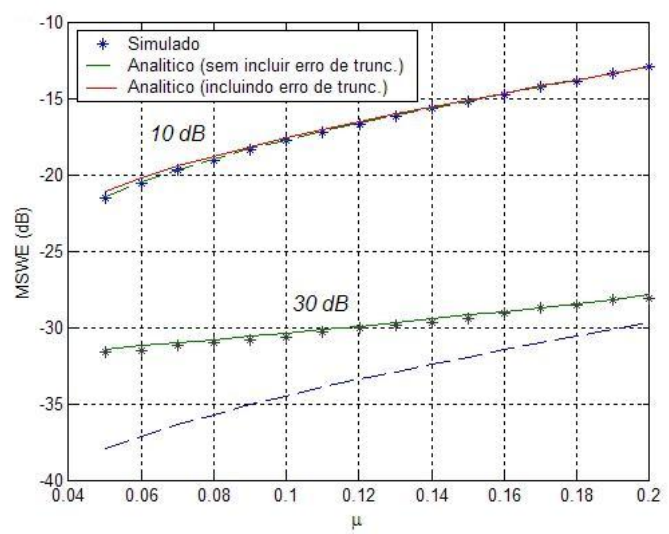

Fig. 3. MSWE em regime permanente em função do passo do algoritmo LMS, obtido por análise e simulação. Canal ITU-R bom, $E_{b} / N_{0}$ de 10 e 30 $\mathrm{dB}$.

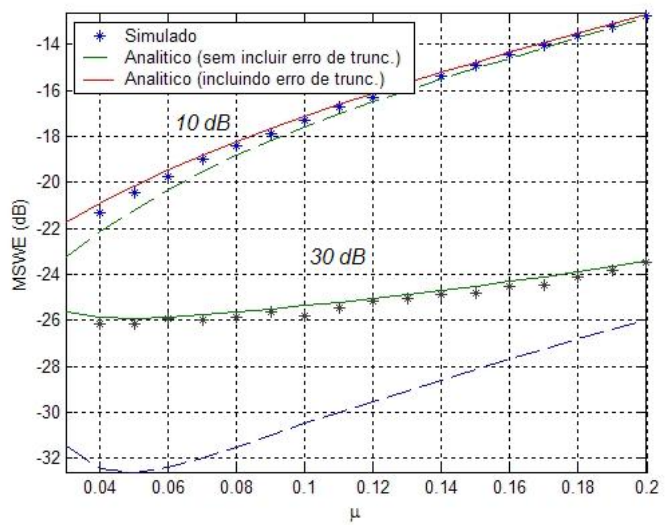

Fig. 4. MSWE em regime permanente em função do passo do algoritmo LMS, obtido por análise e simulação. Canal ITU-R moderado, $E_{b} / N_{0}$ de 10 e $30 \mathrm{~dB}$.

\section{RESUltados NuMÉRICOS}

Dois conjuntos de resultados são apresentados e discutidos, procurando-se validar os resultados analíticos apresentados na Seção III por meio de simulação computacional.

A taxa de símbolos $R_{s}$ utilizada foi de 2400 bauds e empregou-se um esquema de modulação Quadrature Phase Shift Keying (QPSK). O fator de roll-off dos filtros de transmissão e de recepção foi fixado em 0,5 .

\section{A. MSWE em Regime Permanente}

Os resultados apresentados nas três figuras que se seguem foram obtidos a partir de 1000 realizações estatisticamente independentes de todos os processos estocásticos envolvidos na modelagem, utilizando blocos de símbolos transmitidos de comprimento 600. Foram considerados dois valores da razão $E_{b} / N_{0}$ (energia por bit sobre densidade espectral de potência do ruído, na entrada do receptor ): 10 e $30 \mathrm{~dB}$.

Cada uma destas figuras se refere a um tipo de modelo de canal da norma ITU-R F.520. No estimador de canal, foram utilizados 5, 5 e 7 coeficientes para identificar os canais ITU-R bom, moderado e pobre, respectivamente.

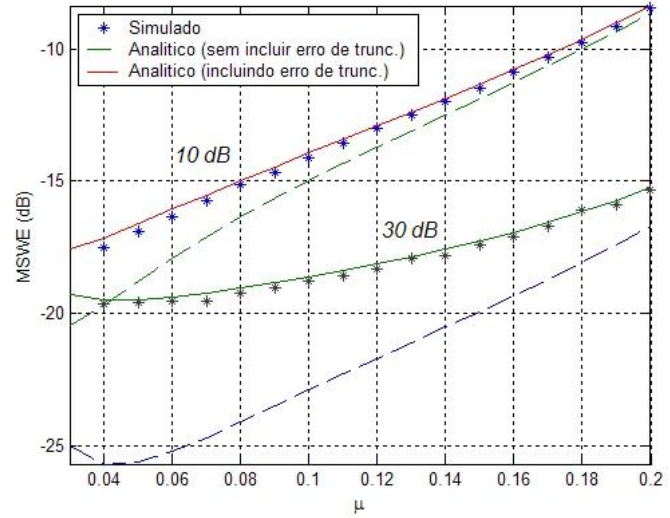

Fig. 5. MSWE em regime permanente em função do passo do algoritmo LMS, obtido por análise e simulação. Canal ITU-R pobre, $E_{b} / N_{0}$ de 10 e $30 \mathrm{~dB}$.

A Figura 3 ilustra os valores analíticos e empíricos de MSWE em regime permanente para o modelo de canal ITU$\mathrm{R}$ bom. As curvas contínuas representam valores analíticos de MSWE obtidos com a inclusão do erro de truncamento, enquanto as curvas tracejadas indicam valores levantados sem considerar este erro. Nesta figura fica evidenciado o impacto do erro de truncamento no MSWE.

A Figura 4 mostra os valores obtidos com o modelo de canal ITU-R moderado. Observa-se nela a ocorrência de degradação de desempenho em relação ao modelo de canal ITU-R bom, em virtude do aumento do espalhamento Doppler, que leva a uma maior dificuldade de rastreamento dos parâmetros do canal.

A Figura 5 exibe os resultados obtidos com o modelo de canal ITU-R pobre. Verifica-se que o efeito da inclusão do erro de truncamento no cálculo do MSWE é mais intenso neste caso. Isto se deve ao maior valor do retardo entre os raios, em relação aos outros dois modelos (ITU-R bom e ITU-R moderado), que leva a um maior erro de truncamento, mesmo com o aumento (de 5 para 7) no número de coeficientes do modelo de canal em tempo discreto.

Por fim, pode-se observar nestas figuras que a inclusão do erro de truncamento na análise do MSWE produziu um excelente nível de aproximação entre valores empíricos e analíticos, em todos os casos considerados.

\section{B. Passo Ótimo Aproximado}

As Figuras 6, 7 e 8 ilustram os valores analíticos de MSWE em regime permanente para cada tipo de modelo de canal de HF da ITU-R F.520, considerando valores de $E_{b} / N_{0}$ iguais a 10,20 e $30 \mathrm{~dB}$. Os pontos correspondentes aos valores do passo ótimo aproximado são destacados com asteriscos.

Percebe-se nestas figuras, como esperado, que o valor de passo que minimiza o MSWE é diferente para cada modelo de canal. Quanto maior é o valor do parâmetro $B_{d}$, maior é a velocidade de variação do canal e, conseqüentemente, maior deve ser o valor de passo a fim de rastrear melhor esta variação.

Observa-se ainda em todos os casos que os valores de passo ótimo aproximado são um pouco maiores que os valores 


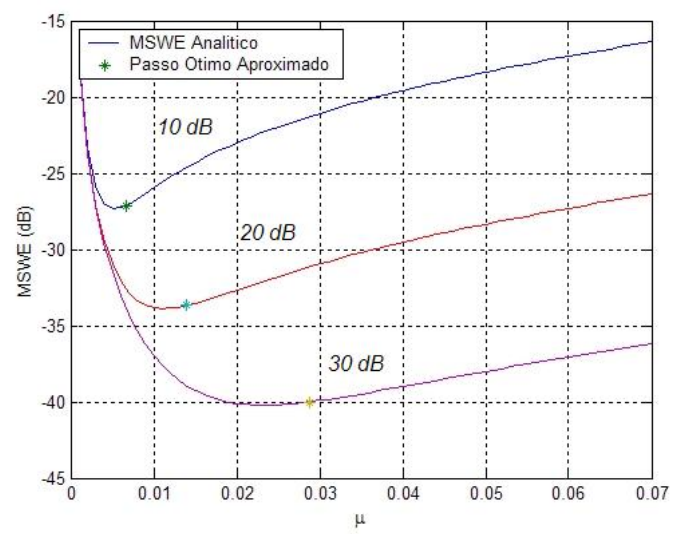

Fig. 6. MSWE em regime permanente em função do passo, obtido analiticamente. Canal ITU-R bom, com $E_{b} / N_{0}$ de 10,20 e $30 \mathrm{~dB}$. Em destaque os valores de MSWE para o passo ótimo aproximado.

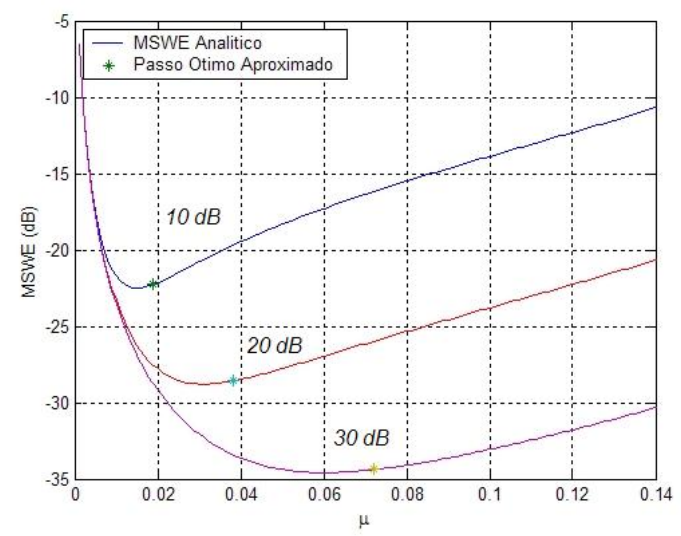

Fig. 7. MSWE em regime permanente em função do passo, obtido analiticamente. Canal ITU-R moderado, com $E_{b} / N_{0}$ de 10,20 e $30 \mathrm{~dB}$ Em destaque os valores de MSWE para o passo ótimo aproximado.

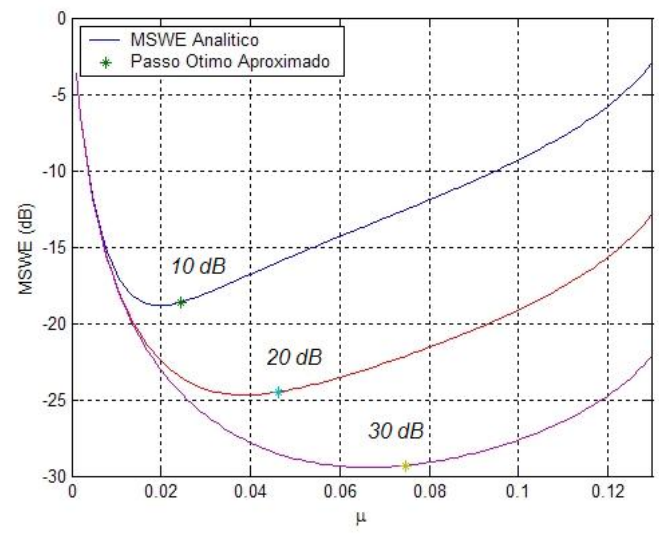

Fig. 8. MSWE em regime permanente obtidas analiticamente para o modelo de canal ITU-R pobre e $E_{b} / N_{0}$ de 10,20 e $30 \mathrm{~dB}$. Em destaque os valores de MSWE para o passo ótimo aproximado. efetivos de passo ótimo, que se pode inferir das curvas obtidas por simulação.

Estas diferenças, além de pequenas, podem ser vistas como um sub-produto interessante da análise aqui apresentada, pois o uso dos valores de passo ótimo aproximados permite obter maiores velocidades de convergência sem provocar aumentos consideráveis no MSWE em regime permanente, como se pode depreender das mesmas figuras.

\section{CONClusões}

Estendeu-se neste artigo a análise desempenho do algoritmo LMS na estimação de canais apresentada em [1], considerando-se aqui canais em HF. Obteve-se uma expressão analítica aproximada para o valor ótimo do passo de adaptação, no sentido da minimização do erro quadrático médio na estimação dos coeficientes (MSWE) em regime estacionário.

Analisou-se também a aplicação do algoritmo LMS na estimação dos coeficientes de um filtro FIR utilizado como modelo em tempo discreto para o sistema composto pelo canal de propagação, filtros de transmissão e recepção, e por um amostrador à taxa de símbolos. Particular atenção foi dada ao efeito sobre o MSWE da limitação do número de coeficientas deste modelo, para fins de simplificação dos receptores que operam com base em estimação do canal.

Foram apresentados diversos resultados numéricos de avaliação de desempenho, obtidos por simulação e via expressões analíticas, utilizando-se modelos de canais da norma ITUR F.520. Estes resultados mostram claramente a validade e utilidade das análises desenvolvidas, tendo sido observado um ajuste muito bom entre análise e simulação.

Verificou-se também que a aproximação analítica aqui desenvolvida para o passo ótimo tende a produzir um valor ligeiramente maior que o efetivo valor de passo ótimo, permitindo obter maior velocidade de convergência do estimador do canal, praticamente sem aumento do MSWE em regime permanente.

Cabe notar ainda que as análises aqui desenvolvidas podem ser facilmente aplicadas a modelos de canal WSS-US utilizados em outros sistemas de comunicações de interesse atual, o que será considerado em trabalhos futuros.

\section{REFERÊNCIAS}

[1] Galdino, J.F., Pinto E. L. e Alencar, M. S. "Analytical performance of the LMS algorithm on estimation of wide sense stationary channels". IEEE Transactions on Communicatons, 2004.

[2] Watterson, C. C. e Bensema,W. D. "Experimental confirmation of an HF channel model". IEEE Trans. on Communication Technology, 1970.

[3] Otnes, R. Bauer, N. "Evaluation of turbo equalization for the high-rate HF waveforms of STANAG 4539". Proc. of the Ninth International Conference on HF Radio Systems and Techniques, 2003, pp. 114-119, June 2003.

[4] Li, Z. and Mämmelä, A. "An Adaptive RSSE-PSP Receiver with a Pre-filter for EDGE Systems". Proc. of the 2003 IEEE International Conference on Communications (ICC'03), vol. 5, pp 1252 - 1256.

[5] Diniz, P. S. R. "Adaptive Filtering - Algorithms and Practical Implementation". Kluwer Academic Publishers, 2002.

[6] Santos, Claudio Canto dos. "Estimação de Canais de HF Usando o Algoritmo LMS para Aplicação em Equalização DFE”. Dissertação de Mestrado - Instituto Militar de Engenharia (IME), 2007.

[7] Pinto, E. L. e Maia, M. A. G. M. "A model for the oversampled received signal in a wss-us transmission environment". Proc. of the 2nd Conference on Telecommunications (CONFTELE99), pp. 137-141, Sesimbra, Portugal, 1999. 